\title{
Linear Control Problems of the Fuzzy Maps
}

\author{
Andrej V. Plotnikov, Tatyana A. Komleva, Irina V. Molchanyuk
}

Department of Applied Mathematics, Odessa State Academy of Civil Engineering and Architecture, Odessa, Ukraine. Email: a-plotnikov@ukr.net, t-komleva@ukr.net,i-molchanuyk@ukr.net

Received October $28^{\text {th }}, 2009$; revised November $16^{\text {th }}, 2009$; accepted November $20^{\text {th }}, 2009$.

\begin{abstract}
In the present paper, we show the some properties of the fuzzy R-solution of the control linear fuzzy differential inclusions and research the optimal time problems for it.
\end{abstract}

Keywords: Fuzzy Differential Inclusions, Control Problems

\section{Introduction}

The first study of differential equations with multivalued right-hand sides was performed by A. Marchaud [1] and S. C. Zaremba [2]. In early sixties, T. Wazewski [3,4], A. F. Filippov [5] obtained fundamental results on existence and properties of the differential equations with multivalued right-hand sides (differential inclusions). One of the most important results of these articles was an establishment of the relation between differential inclusions and optimal control problems, that promoted to develop the differential inclusion theory [6-9].

Considering of the differential inclusions required to study properties of multivalued functions, i.e. an elaboration the whole tool of mathematical analysis for multivalued functions $[6,10,11]$.

In works [12,13] annotate of an R-solution for differential inclusion is introduced as an absolutely continuous multivalued function. Various problems for the R-solution theory were regarded in [14-18]. The basic idea for a development of an equation for R-solutions (integral funnels) is contained in [19].

In the last years there has been forming new approach to control problems of dynamic systems, which foundation on analysis of trajectory bundle but not separate trajectories. The section of this bundle in any instant is some set and it is necessary to describe the evolution of this set. Obtaining and research dynamic equations of sets there is important problem in this case. The metric space of sets with the Hausdorff metric is natural space for description dynamic of sets. In theory of multivalued maps definitions on derivative as for single-valued maps is impossible because space of sets is nonlinear. This bound possibility description dynamic sets by differential equations. Therefore, the control differential equations with set of initial conditions [20-22] and the control differential inclusions [8,23-34] use for it.

In recent years, the fuzzy set theory introduced by Zadeh [35] has emerged as an interesting and fascinating branch of pure and applied sciences. The applications of fuzzy set theory can be found in many branches of regional, physical, mathematical, differential equations, and engineering sciences. Recently there have been new advances in the theory of fuzzy differential equations [36-47] and inclusions [43,48-52] as well as in the theory of control fuzzy differential equations [53-55] and inclusions [56,57].

In this article we consider the some properties of the fuzzy R-solution of the control linear fuzzy differential inclusions and research the optimal time problems for it.

\section{The Fundamental Definitions and Designations}

Let $\operatorname{comp}\left(R^{n}\right)\left(\operatorname{conv}\left(R^{n}\right)\right)$ be a set of all nonempty (convex) compact subsets from the space $R^{n}$,

$$
h(A, B)=\min _{r \geq 0}\left\{S_{r}(A) \supset B, S_{r}(B) \supset A\right\}
$$

be Hausdorff distance between sets $A$ and $B, S_{r}(A)$ is $r$-neighborhood of set $A$.

Let $E^{n}$ be the set of all $u: R^{n} \rightarrow[0,1]$ such that $u$ satisfies the following conditions:

1) $u$ is normal, that is, there exists an $x_{0} \in R^{n}$ such that $u\left(x_{0}\right)=1$;

2) $u$ is fuzzy convex, that is,

$$
u(\lambda x+(1-\lambda) y) \geq \min \{u(x), u(y)\}
$$

3) For any $x, y \in R^{n}$ and $0 \leq \lambda \leq 1$;

4) $u$ is upper semicontinuous; 
5) $[u]^{0}=\operatorname{cl}\left\{x \in R^{n}: u(x)>0\right\}$ is compact.

If $u \in E^{n}$, then $u$ is called a fuzzy number, and $E^{n}$ is said to be a fuzzy number space. For $0<\alpha \leq 1$, denote

$$
[u]^{\alpha}=\left\{x \in R^{n}: u(x) \geq \alpha\right\} .
$$

Then from 1)-4), it follows that the $\alpha$-level set $[u]^{\alpha} \in \operatorname{conv}\left(R^{n}\right)$ for all $0 \leq \alpha \leq 1$.

Theorem 1. (Negoita and Ralescu [58]). If $u \in E^{n}$, then

1) $[u]^{\alpha} \in \operatorname{conv}\left(R^{n}\right)$ for all $\alpha \in[0,1]$;

2) $[u]^{\alpha} \subset[u]^{\beta}$ for $0 \leq \alpha<\beta \leq 1$;

3) If $\left\{\alpha_{k}\right\} \subset[0,1]$ is a decreasing sequence converging to $\alpha>0$ then

$$
[u]^{\alpha}=\bigcap_{k \geq 1}[u]^{\alpha k}
$$

Conversely, if $\left\{A^{\alpha}: 0 \leq \alpha \leq 1\right\}$ is a family of convex compact subsets of $R^{n}$ satisfying 1)-3), then $[u]^{\alpha}=A^{\alpha}$ for $0<\alpha \leq 1$ and

$$
[u]^{0}=\overline{\bigcap_{0<\alpha \leq 1} A^{\alpha}} \subset A^{0} .
$$

If $g: R^{n} \times R^{n} \rightarrow R^{n}$ is a function, then using Zadeh's extension principle we can extend $\widetilde{g}$ to $E^{n} \times E^{n} \rightarrow E^{n}$ by the equation

$$
\tilde{g}(u, v)(z)=\sup _{z=g(x, y)} \min \{u(x), v(y)\} .
$$

It is well known that

$$
[\widetilde{g}(u, v)]^{\alpha}=g\left([u]^{\alpha},[v]^{\alpha}\right)
$$

for all $u, v \in E^{n}, 0 \leq \alpha \leq 1$ and continuous function $g$. Further, we have

$$
[u+v]^{\alpha}=[u]^{\alpha}+[v]^{\alpha},[k u]^{\alpha}=k[u]^{\alpha},
$$

where $k \in R$.

Define $D: E^{n} \times E^{n} \rightarrow[0, \infty)$ by the relation

$$
D(u, v)=\sup _{0 \leq \alpha \leq 1} h\left([u]^{\alpha},[v]^{\alpha}\right),
$$

where $h$ is the Hausdorff metric defined in $\operatorname{comp}\left(R^{n}\right)$. Then $D$ is a metric in $E^{n}$.

Further we know that [59]

1) $\left(E^{n}, D\right)$ is a complete metric space;

2) $D(u+w, v+w)=D(u, v)$ for all $u, v, w \in E^{n}$;

3) $D(\lambda u, \lambda v)=|\lambda| D(u, v)$ for all $u, v \in E^{n}$ and $\lambda \in R$.

It can be proved that

$$
D(u+v, w+z) \leq D(u, w)+D(v, z)
$$

for $u, v, w, z \in E^{n}$.

Definition 1. A mapping $F:[0, T] \rightarrow E^{n}$ is strongly measurable if for all $\alpha \in[0,1]$ the set-valued map $F_{\alpha}:[0, T] \rightarrow \operatorname{conv}\left(R^{n}\right)$ defined by $F_{\alpha}(t)=[F(t)]^{\alpha}$ is Lebesgue measurable.

Definition 2. A mapping $F:[0, T] \rightarrow E^{n}$ is said to be integrably bounded if there is an integrable function $h(t)$ such that $\|x(t)\| \leq h(t)$ for every $x(t) \in F_{0}(t)$.

Definition 3. The integral of a fuzzy mapping $F:[0, T] \rightarrow E^{n}$ is defined levelwise by $\left[\int_{0}^{T} F(t) d t\right]^{\alpha}$ $=\int_{0}^{T} F_{\alpha}(t) d t$ The set of all $\int_{0}^{T} f(t) d t$ such that $f:[0, T] \rightarrow R^{n}$ is a measurable selection for $F_{\alpha}$ for all $\alpha \in[0,1]$.

Definition 4. A strongly measurable and integrably bounded mapping $F:[0, T] \rightarrow E^{n}$ is said to be integrable over $[0, T]$ if $\int_{0}^{T} F(t) d t \in E^{n}$.

Note that if $F:[0, T] \rightarrow E^{n}$ is strongly measurable and integrably bounded, then $F$ is integrable. Further if $F:[0, T] \rightarrow E^{n}$ is continuous, then it is integrable.

Theorem 2. [36]. Let $F, G:[0, T] \rightarrow E^{n}$ be integrable and $c \in[0, T], \lambda \in R$. Then

1) $\int_{0}^{T} F(t) d t=\int_{0}^{c} F(t) d t+\int_{c}^{T} F(t) d t$

2) $\int_{0}^{T} F(t)+G(t) d t=\int_{0}^{T} F(t) d t+\int_{0}^{T} G(t) d t$;

3) $\int_{0}^{T} \lambda F(t) d t=\lambda \int_{0}^{T} F(t) d t$;

4) $D(F, G)$ is integrable;

5) $D\left(\int_{0}^{T} F(t) d t, \int_{0}^{T} G(t) d t\right) \leq \int_{0}^{T} D(F(t), G(t)) d t$

Consider the following control linear fuzzy differential inclusions

$$
\dot{x} \in A(t) x+G(t, w), x\left(t_{0}\right)=x_{0},
$$

and the following nonlinear fuzzy differential inclusions

$$
\dot{x} \in F(t, x, w), \quad x\left(t_{0}\right)=x_{0},
$$

where $\dot{x}$ means $d x / d t ; t \in R_{+}$is the time; $x \in R^{n}$ is the state; $w \in R^{m}$ is the control; $A(t)$ is $(n \times n)$-dimensional matrix-valued function; $G: R_{+} \times R^{m} \rightarrow E^{n}$, $F: R_{+} \times R^{n} \times R^{m} \rightarrow E^{n}$ are the set-valued functions.

Let 


$$
W: R_{+} \rightarrow \operatorname{conv}\left(R^{m}\right)
$$

be the measurable multivalued map.

Definition 5. Set $L W$ of all single-valued branches of the multivalued map $W($.$) is the set of the possible$ controls.

Obviously, the control fuzzy differential Inclusion (2) turns into the ordinary fuzzy differential inclusion

$$
\dot{x} \in \Phi(t, x), \quad x\left(t_{0}\right)=x_{0},
$$

if the control $\widetilde{w}(\cdot) \in L W$ is fixed and $\Phi(t, x) \equiv$ $F(t, x, \widetilde{w}(t))$.

The fuzzy differential Inclusions (3) has the fuzzy R-solution, if right-hand side of the fuzzy differential Inclusion (3) satisfies some conditions [52].

Let $X(t)$ denotes the fuzzy R-solution of the differential Inclusion (3), then $X(t, w)$ denotes the fuzzy $\mathrm{R}$-solution of the control differential Inclusion (2) for the fixed $w(\cdot) \in L W$.

Definition 6. The set

$$
Y(T)=\{X(T, w): w(\cdot) \in L W\}
$$

be called the attainable set of the fuzzy System (2).

\section{The Some Properties of the R-Solution}

In this section, we consider the some properties of the R-solution of the control fuzzy differential Inclusion (1).

Let the following condition is true.

\section{Condition A:}

A1. $A(\cdot)$ is measurable on $\left[t_{0}, T\right]$;

A2. The norm $\|A(t)\|$ of the matrix $A(t)$ is integrable on $\left[t_{0}, T\right]$;

A3. The multivalued map $W:\left[t_{0}, T\right] \rightarrow \operatorname{conv}\left(R^{m}\right)$ is measurable on $\left[t_{0}, T\right]$;

A4. The fuzzy map $G: R_{+} \times R^{m} \rightarrow E^{n}$ satisfies the conditions

1) measurable in $t$

2) continuous in $w$;

A5. There exist $v(\cdot) \in L_{2}\left[t_{0}, T\right]$ and $l(\cdot) \in L_{2}\left[t_{0}, T\right]$ such that

$$
|W(t)| \leq v(t), \quad|G(t, w)| \leq l(t)
$$

almost everywhere on $\left[t_{0}, T\right]$;

A6. The set $Q(t)=\{G(t, w(t)): w(\cdot) \in L W\}$ is compact and convex for almost every $\left[t_{0}, T\right]$, i.e. $Q(t) \in \operatorname{conv}\left(E^{n}\right)$.

Theorem 3. Let the condition A is true.

Then for every $w(\cdot) \in L W$ there exists the fuzzy R-solution $X(\cdot, w)$ such that
1) the fuzzy map $X(\cdot, w)$ has form

$$
X(t, w)=\Phi(t) x_{0}+\Phi(t) \int_{t_{0}}^{t} \Phi^{-1}(s) G(s, w(s)) d s,
$$

where $t \in\left[t_{0}, T\right] ; \Phi(t)$ is Cauchy matrix of the differential equation $\dot{x}=A(t) x$;

2) $X(t, w) \in E^{n}$ for every $t \in\left[t_{0}, T\right]$;

3) the fuzzy map $X(\cdot, w)$ is the absolutely continuous fuzzy map on $\left[t_{0}, T\right]$.

Proof. The proof is easy consequence of the $[31,34,52,54]$ and Theorem 1 .

Theorem 4. Let the condition A is true.

Then the attainable set $Y(T)$ is compact and convex.

Proof. The proof is easy consequence of the $[31,34,52,54]$ and Theorem 1 .

We obtained the basic properties of the fuzzy R-solution of System (1). Now, we consider the some control fuzzy problems.

\section{The Optimal Time Problems}

Consider the control linear fuzzy differential Inclusion (1), when

$$
G(t, w)=B(t) w+F(t)
$$

where

B1. $B(\cdot)$ is measurable on $\left[t_{0}, T\right]$;

B2. The norm $\|B(t)\|$ of the matrix $B(t)$ is integrable on $\left[t_{0}, T\right]$;

B3. The fuzzy map $F:\left[t_{0}, T\right] \rightarrow E^{n}$ is measurable on $\left[t_{0}, T\right]$;

B4. There exists $f(\cdot) \in L_{2}\left[t_{0}, T\right]$ such that

$$
|F(t)| \leq f(t)
$$

almost everywhere on $\left[t_{0}, T\right]$.

Consider the following optimal control problem: it is necessary to find the minimal time $T$ and the control $w^{*}(.) \in L W$ such that the fuzzy R-solution of Systems (1),(4) satisfies one of the conditions:

$$
\begin{aligned}
& X\left(T, w^{*}\right) \cap S_{k} \neq \varnothing, \\
& X\left(T, w^{*}\right) \subset S_{k}, \\
& X\left(T, w^{*}\right) \supset S_{k},
\end{aligned}
$$

where $S_{k} \in E^{n}$ is the terminal set.

Clearly, these time optimal problems are different from the ordinary time optimal problem by that here control object has the volume.

Definition 6. We shall say that the pair $\left(w^{*}(\cdot), X\left(\cdot, w^{*}\right)\right)$ satisfies the maximum principle on $\left[t_{0}, T\right]$, if there exists the vector-function $\psi(\cdot)$, which is the solution of the 
system

$$
\dot{\psi} \in-A^{T}(t) \psi, \psi(T) \in S_{1}(0)
$$

and the following conditions are true

1) the maximum condition

$$
C\left(B(t) w^{*}(t), \psi(t)\right)=\max _{w \in W(t)} C(B(t) w, \psi(t))
$$

almost everywhere on $\left[t_{0}, T\right]$;

2) the transversal condition:

a) in the case (5):

$$
C\left(\left[X\left(T, w^{*}\right)\right]^{1}, \psi(T)\right)=-C\left(\left[S_{k}\right]^{1},-\psi(T)\right) ;
$$

b) in the case (6): for all $\alpha \in[0,1]$

$$
C\left(\left[X\left(T, w^{*}\right)\right]^{\alpha}, \psi(T)\right) \leq C\left(\left[S_{k}\right]^{\alpha}, \psi(T)\right)
$$

and there exists $\beta \in[0,1]$ such that

$$
C\left(\left[X\left(T, w^{*}\right)\right]^{\beta}, \psi(T)\right)=C\left(\left[S_{k}\right]^{\beta}, \psi(T)\right) ;
$$

c) in the case (6): for all $\beta \in[0,1]$

$$
C\left(\left[X\left(T, w^{*}\right)\right]^{\alpha},-\psi(T)\right) \leq C\left(\left[S_{k}\right]^{\alpha},-\psi(T)\right)
$$

and there exists $\beta \in[0,1]$ such that

$$
C\left(\left[X\left(T, w^{*}\right)\right]^{\beta},-\psi(T)\right)=C\left(\left[S_{k}\right]^{\beta},-\psi(T)\right) .
$$

Clearly, that there cases of the transversal condition of the maximum principle correspond to the three cases of the time optimal problems.

Theorem 5. (necessary optimal condition). Let the condition A are true and the pair $\left(T, w^{*}(\cdot)\right)$ is optimality.

Then the pair $\left(w^{*}(\cdot), X\left(\cdot, w^{*}\right)\right)$ satisfies the maximum principle on $\left[t_{0}, T\right]$.

Proof. Let $w^{*}(\cdot)$ is the optimal control and $X\left(\cdot, w^{*}\right)$ is the optimal R-solution of the Systems (1),(4), i.e.

1) $X\left(T, w^{*}\right) \in Y(T)$;

2) $X\left(T, w^{*}\right) \cap S_{k}=\varnothing$.

From 1) and 2) we have

$$
\max _{X \in[Y(T)]^{1}} C(X, \psi) \geq C\left(\left[S_{k}\right]^{1},-\psi\right)
$$

for all $\psi \in S_{1}(0)$.

Consequently

$$
p=\max _{X \in[Y(T)]^{1}} \min _{\psi \in S_{1}(0)} C(X, \psi)+C\left(\left[S_{k}\right]^{1},-\psi\right) \geq 0 .
$$

From $\left[X\left(T, w^{*}\right)\right]^{1} \cap\left[S_{k}\right]^{1} \neq \varnothing$ we have

$$
q(T, \psi)=C\left(\left[X\left(T, w^{*}\right)\right]^{1}, \psi\right)+C\left(\left[S_{k}\right]^{1},-\psi\right) \geq 0
$$

for all $\psi \in S_{1}(0)$.

From Theorem 1 we have that the function $q(T, \psi)$ is continuous on $R_{+} \times S_{1}(0)$.

If $q(T, \psi)>0$ for all $\psi \in S_{1}(0)$ then we have $q^{0}(T)=\min _{\psi \in S_{1}(0)} q(T, \psi) \geq \gamma>0$. Hence there exists $\tau<T$ such that $q^{0}(\tau) \geq 0$. Consequently we have

$$
C\left(\left[X\left(\tau, w^{*}\right)\right]^{1}, \psi\right)+C\left(\left[S_{k}\right]^{1},-\psi\right) \geq 0
$$

for all $\psi \in S_{1}(0)$, i.e. $\left[X\left(\tau, w^{*}\right)\right]^{1} \cap\left[S_{k}\right]^{1} \neq \varnothing$.

It contradicts that $T$ is optimal time.

If $p>0$,

$$
\begin{aligned}
& \max _{X \in[Y(T)]^{1}} \min _{\psi \in S_{1}(0)} C(X, \psi)+C\left(\left[S_{k}\right]^{1},-\psi\right) \\
& =C(\tilde{X}, \tilde{\psi})+C\left(\left[S_{k}\right]^{1},-\tilde{\psi}\right)
\end{aligned}
$$

and $\left[X\left(T, w^{*}\right)\right]^{\natural} \neq \tilde{X}$, than we have a contradiction. Hence there exist $\tilde{\psi} \in S_{1}(0)$ such that

$$
\begin{aligned}
& C\left(\left[X\left(T, w^{*}\right)\right]^{1}, \tilde{\psi}\right)=\max _{X \in[Y(T)]^{1}} C(X, \tilde{\psi}), \\
& C\left(\left[X\left(T, w^{*}\right)\right]^{1}, \tilde{\psi}\right)=-C\left(\left[S_{k}\right]^{1},-\tilde{\psi}\right) .
\end{aligned}
$$

Consequently

$$
\begin{aligned}
& \left(\int_{0}^{T} \Phi(T) \Phi^{-1}(s) B(s) w^{*}(s) d s, \tilde{\psi}\right) \\
& =\max _{w(\cdot) \in L W}\left(\int_{0}^{T} \Phi(T) \Phi^{-1}(s) B(s) w(s) d s, \tilde{\psi}\right)
\end{aligned}
$$

Then we have

$$
\begin{aligned}
& \left(\Phi(T) \Phi^{-1}(s) B(s) w^{*}(s), \tilde{\psi}\right) \\
& =\max _{w(\cdot) \in L W}\left(\Phi(T) \Phi^{-1}(s) B(s) w(s), \tilde{\psi}\right)
\end{aligned}
$$

for almost everywhere $s \in\left[t_{0}, T\right]$. If

$$
\psi(t)=\left(\Phi(T) \Phi^{-1}(t)\right)^{T} \tilde{\psi} /\left\|\left(\Phi(T) \Phi^{-1}(t)\right)^{T} \tilde{\psi}\right\|,
$$

than the theorem is proved.

Example. Consider the following control linear fuzzy differential inclusions

$$
\dot{x} \in\left(\begin{array}{cc}
0 & 1 \\
-1 & 0
\end{array}\right) x+w+F, x(0)=0,
$$

where $x=\left(x_{1}, x_{2}\right)^{T}$ is the state; $w=\left(w_{1}, w_{2}\right)^{T} \in W=S_{1}(0)$ is the control; $F \in E^{2}$ is the fuzzy set, where 


$$
v(f)=\left\{\begin{array}{ll}
1-4 f_{1}^{2}-9 f_{2}^{2}, & 4 f_{1}^{2}+9 f_{2}^{2} \leq 1 \\
0, & 4 f_{1}^{2}+9 f_{2}^{2}>1
\end{array} .\right.
$$

Consider the following optimal control problem: it is necessary to find the minimal time $T$ and the control $w^{*}(\cdot) \in L W$ such that the fuzzy R-solution of system satisfies of the conditions:

$$
X\left(T, w^{*}\right) \cap S_{k} \neq \varnothing
$$

where $S_{k} \in E^{2}$ is the terminal set such, that

$$
\begin{aligned}
& \sigma(x)= \begin{cases}\sqrt{1-\left(x_{1}-2 \pi\right)^{2}-\left(x_{2}-1\right)^{2}}, & x \in Q, x_{2} \geq 1 \\
\sqrt{1-\left(x_{1}-2 \pi\right)^{2}} & x \in Q,-1<x_{2}<1 \\
\sqrt{1-\left(x_{1}-2 \pi\right)^{2}-\left(x_{2}+1\right)^{2}} & x \in Q, x_{2} \leq-1 \\
0 & x \notin Q\end{cases}
\end{aligned}
$$

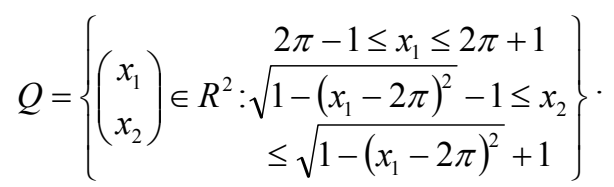

Obviously, the optimal pair $T=2 \pi$ and $w^{*}(t)=$ $(\cos (t),-\sin (t))$ satisfy of the conditions of the Theorem 5:

1) $\left(w^{*}(t), \psi(t)\right)=C(W, \psi(t))$ for a.e. $t \in[0,2 \pi]$;

2) $C\left(\left[X\left(T, w^{*}\right)\right]^{1}, \psi(T)\right)=-C\left(\left[S_{k}\right]^{1},-\psi(T)\right)$,

where $\psi(t)=(\cos (t),-\sin (t))^{T}$ for a.e. $t \in[0,2 \pi]$,

$$
\begin{gathered}
{\left[X\left(T, w^{*}\right)\right]^{1}=(T \cos (T),-T \sin (T))^{T}=(2 \pi, 0)^{T},} \\
{\left[S_{k}\right]^{1}=\left\{\left(x_{1}, x_{2}\right)^{T}: x_{1}=2 \pi,-1 \leq x_{2} \leq 1\right\} .}
\end{gathered}
$$

\section{Conclusions}

In the last decades, a number of works devoted to problems of optimal control of multiple-valued trajectories (fuzzy trajectories, trajectory bundles or an ensemble of trajectories) appeared; these works fall into a subdivision of the optimal control theory, namely, the theory of process control under uncertainty and fuzzy conditions. This is conditioned by the fact that, in actual problems arising in economy and engineering in the course of construction of a mathematical model, it is practically impossible to exactly describe the behavior of an object. This is explained by the following fact. First, for some parameters of the object, it impossible to specify exact values and laws of their change, but it is possible to determine the domain of these changes. Second, for the sake of simplicity of the mathematical model being constructed, the equations that describe the behavior of the object are simplified and one should estimate the conse- quences of such a simplification. Therefore, if is possible to divide the articles devoted to this direction into two types characterized by the following distinctive features:

1) There exists an incomplete or fuzzy information on the initial data;

2) The equations describing the behavior of the object to be controlled are assumed to be inexact, for example, they can contain some parameters whose exact values and laws of variation are unknown but the domain of their values is fuzzy.

In the second case, fuzzy differential inclusions are frequently used to describe behavior of objects. The reason is that, first this approach is most obvious and, second, theory of fuzzy and ordinary differential inclusions is well found and is rapidly developed at the present time.

In the present paper, the necessary conditions of optimal of control for a system of the latter form of equations with the fuzzy R-solutions are formulated and proved.

\section{REFERENCES}

[1] A. Marchaud, "Sur les champs de demicones et equations differentielles du premier order," Bulletin of Mathematical Society, France, No. 62, pp. 1-38, 1934.

[2] S. C. Zaremba, "Sur une extension de la notion d'equation differentielle," Comptes Rendus l'Académie des Sciences, Paris, No. 199, pp. 1278-1280, 1934.

[3] T. Wazewski, "Systemes de commande et equations au contingent," Bulletin L'Académie Polonaise des Science, SSMAP, No. 9, pp. 151-155, 1961.

[4] T. Wazewski, "Sur une condition equivalente e l'equation au contingent," Bulletin L'Académie Polonaise des Science, SSMAP, No. 9, pp. 865-867, 1961.

[5] A. F. Filippov, "Classical solutions of differential equations with multi-valued right-hand side," SIAM Journal of Control, No. 5, pp. 609-621, 1967.

[6] J.-P. Aubin and A. Cellina, "Differential inclusions. Set-valued maps and viability theory," Springer-Verlag, Berlin-Heidelberg-New York-Tokyo, 1984.

[7] N. Kikuchi, "On contingent equations," Japan-United States Seminar on Ordinary Differential and Functional Equations, Lecture Notes in Mathematics, Springer, Berlin, Vol. 243, pp. 169-181, 1971.

[8] V. A. Plotnikov, A. V. Plotnikov, and A. N. Vityuk, "Differential equations with multivalued right-hand sides," Asymptotics Methods, AstroPrint, Odessa, 1999.

[9] G. V. Smirnov, "Introduction to the theory of differential inclusions," Graduate Studies in Mathematics, American Mathematical Society, Providence, Rhode Island, Vol. 41, 2002.

[10] J.-P. Aubin and H. Frankovska, "Set-valued analysis," Birkhauser, Systems and Control: Fundations and Applications, 1990.

[11] F. S. de Blasi and F. IerVolino, "Equazioni differentialicon soluzioni a valore compatto convesso," Bollettino 
della Unione Matematica Italiana, Vol. 2, No. 4-5, pp. 491-501, 1969.

[12] A. I. Panasyuk, "Dynamics of sets defined by differential inclusions," Siberian Mathematical Journal, Vol. 27, No. 5, pp. 155-165, 1986.

[13] A. I. Panasyuk, "On the equation of an integral funnel and its applications," Differential Equations, Vol. 24, No. 11, pp. 1263-1271, 1988.

[14] A. I. Panasyuk, "Equations of attainable set dynamics, part 1: Integral funnel equations," Journal of Optimization Theory and Applications, Vol. 64, No. 2, pp. 349-366, 1990. "Equations of attainable set dynamics part 2: Partial differential equations," Journal of Optimization Theory and Applications, Vol. 64, No. 2, pp. 367-377, 1990.

[15] A. I. Panasyuk and V. I. Panasyuk, "Asymptotic optimization of nonlinear control systems," Izdatel Belorussia Gosudarstvo University, Minsk, 1977.

[16] A. I. Panasjuk and V. I. Panasjuk, "An equation generated by a differential inclusion," Matematicheskie Zametki, Vol. 27, No. 3, pp. 429-437, 1980.

[17] A. I. Panasyuk and V. I. Panasyuk, "Asymptotic turnpike optimization of control systems," Nauka i Tekhnika, Minsk, 1986.

[18] A. A. Tolstonogov, "On an equation of an integral funnel of a differential inclusion," Matematicheskie Zametki, Vol. 32, No. 6, pp. 841-852, 1982.

[19] A. I. Panasyuk, "Quasidifferential equations in a metric space," Differentsial'nye Uravneniya, Vol. 21, No. 8, pp. 1344-1353, 1985.

[20] D. A. Ovsyannikov, "Mathematical methods for the control of beams," Leningrad University, Leningrad, 1980.

[21] V. I. Zubov, "Dynamics of controlled systems," Vyssh. Shkola, Moscow, 1982.

[22] V. I. Zubov, "Stability of motion: Lyapunov methods and their application," Vyssh. Shkola, Moscow, 1984.

[23] S. Otakulov, "A minimax control problem for differential inclusions," Soviet Doklady Mathematics, Vol. 36, No. 2, pp. 382-387, 1988.

[24] S. Otakulov, "Approximation of the optimal-time problem for controlled differential inclusions," Cybernetics Systems Analysis, Vol. 30, No. 3, pp. 458-462, 1994.

[25] A. V. Plotnikov, "Linear control systems with multivalued trajectories," Kibernetika, Kiev, No. 4, pp. 130-131, 1987.

[26] A. V. Plotnikov, "Compactness of the attainability set of a nonlinear differential inclusion that contains a control," Kibernetika, Kiev, No. 6, pp. 116-118, 1990.

[27] A. V. Plotnikov, "A problem on the control of pencils of trajectories," Siberian Mathematical Journal, Vol. 33, No. 2, pp. 351-354, 1992.

[28] A. V. Plotnikov, "Two control problems under uncertainty conditions," Cybernet Systems Analysis, Vol. 29, No. 4, pp. 567-573, 1993.

[29] A. V. Plotnikov, "Controlled quasi-differential equations and some of their properties," Differential Equations, Vol. 34, No. 10, pp. 1332-1336, 1998.
[30] A. V. Plotnikov, "Necessary optimality conditions for a nonlinear problems of control of trajectory bundles," Cybernetics and System Analysis, Vol. 36, No. 5, pp. 729-733, 2000.

[31] A. V. Plotnikov, "Linear problems of optimal control of multiple-valued trajectories," Cybernetics and System Analysis, Vol. 38, No. 5, pp. 772-782, 2002.

[32] A. V. Plotnikov and T. A. Komleva, "Some properties of trajectory bunches of controlled bilinear inclusion," Ukrainian Mathematical Journal, Vol. 56, No. 4, pp. 586600, 2004.

[33] A. V. Plotnikov and L. I. Plotnikova, "Two problems of encounter under conditions of uncertainty," Journal of Applied Mathematics and Mechanics, Vol. 55, No. 5, pp. 618-625, 1991.

[34] V. A. Plotnikov and A. V. Plotnikov, "Multivalued differential equations and optimal control," Applications of Mathematics in Engineering and Economics, Heron Press, Sofia, pp. 60-67, 2001.

[35] L. A. Zadeh, "Fuzzy sets," Information and Control, No. 8, pp. 338-353, 1965.

[36] O. Kaleva, "Fuzzy differential equations," Fuzzy Sets and Systems, Vol. 24, No. 3, pp. 301-317, 1987.

[37] O. Kaleva, "The Cauchy problem for fuzzy differential equations," Fuzzy Sets and Systems, Vol. 35, No. 3, pp. 389-396, 1990.

[38] O. Kaleva, "The Peano theorem for fuzzy differential equations revisited," Fuzzy Sets and Systems, Vol. 98, No. 1, pp. 147-148, 1998.

[39] O. Kaleva, "A note on fuzzy differential equations," Nonlinear Analysis, Vol. 64, No. 5, pp. 895-900, 2006.

[40] T. A. Komleva, L. I. Plotnikova, and A. V. Plotnikov, "Averaging of the fuzzy differential equations," Work of the Odessa Polytechnical University, Vol. 27, No. 1, pp. 185-190, 2007.

[41] T. A. Komleva, A. V. Plotnikov, and N. V. Skripnik, "Differential equations with set-valued solutions," Ukrainian Mathematical Journal, Springer, New York, Vol. 60, No. 10, pp. 1540-1556, 2008.

[42] V. Lakshmikantham, T. G. Bhaskar, and D. J. Vasundhara, "Theory of set differential equations in metric spaces," Cambridge Scientific Publishers, Cambridge, 2006.

[43] V. Lakshmikantham and R. N. Mohapatra, "Theory of fuzzy differential equations and inclusions," Series in Mathematical Analysis and Applications, Taylor \& Francis Ltd., London, Vol. 6, 2003.

[44] J. Y. Park and H. K. Han, "Existence and uniqueness theorem for a solution of fuzzy differential equations," International Journal of Mathematics and Mathematical Sciences, Vol. 22, No. 2, pp. 271-279, 1999.

[45] J. Y. Park and H. K. Han, "Fuzzy differential equations," Fuzzy Sets and Systems, Vol. 110, No. 1, pp. 69-77, 2000.

[46] S. Seikkala, "On the fuzzy initial value problem," Fuzzy Sets and Systems, Vol. 24, No. 3, pp. 319-330, 1987.

[47] D. Vorobiev and S. Seikkala, "Towards the theory of fuzzy differential equations," Fuzzy Sets and Systems, 
Vol. 125, No. 2, pp. 231-237, 2002.

[48] J.-P. Aubin, "Mutational equations in metric spaces," Set-Valued Analysis, Vol. 1, No. 1, pp. 3-46, 1993.

[49] J.-P. Aubin, "Fuzzy differential inclusions," Problems of Control and Information Theory, Vol. 19, No. 1, pp. 5567, 1990.

[50] V. A. Baidosov, "Differential inclusions with fuzzy righthand side," Soviet Mathematics, Vol. 40, No. 3, pp. 567-569, 1990 .

[51] V. A. Baidosov, "Fuzzy differential inclusions," Journal of Applied Mathematics and Mechanics, Vol. 54, No. 1, pp. 8-13, 1990.

[52] E. Hullermeier, "An approach to modeling and simulation of uncertain dynamical systems," International Journal of Uncertainty, Fuzziness Knowledge-Based Systems, Vol. 5, No. 2, pp. 117-137, 1997.

[53] N. D. Phu and T. T. Tung, "Some properties of sheafsolutions of sheaf fuzzy control problems," Electronic Journal of Differential Equations, No. 108, pp. 1-8, 2006. http://www.ejde.math.txstate.edu.

[54] N. D. Phu and T. T. Tung, "Some results on sheaf-solutions of sheaf set control problems," Nonlinear Analysis, Vol. 67, No. 5, pp. 1309-1315, 2007.

[55] N. D. Phu and T. T. Tung, "Existence of solutions of fuzzy control differential equations," Journal of Sci-Tech Development, Vol. 10, No. 5, pp. 5-12, 2007.

[56] I. V. Molchanyuk and A. V. Plotnikov, "Linear control systems with a fuzzy parameter," Nonlinear Oscillator, Vol. 9, No. 1, pp. 59-64, 2006.

[57] V. S. Vasil'kovskaya and A. V. Plotnikov, "Integrodifferential systems with fuzzy noise," Ukrainian Mathematical Journal, Vol. 59, No. 10, pp. 1482-1492, 2007.

[58] C. V. Negoito and D. A. Ralescu, "Applications of fuzzy sets to systems analysis," A Halsted Press Book, John Wiley \& Sons, New York-Toronto, Ont., 1975.

[59] M. L. Puri and D. A. Ralescu, "Fuzzy random variables," Journal of Mathematical Analysis and Applications, No. 114, pp. 409-422, 1986. 